Supporting Information

\title{
NIR-triggered Dynamic Wrinkling Biointerface for Noninvasive Harvesting of Practical Cell Sheets
}

Wei Xu, ${ }^{1}$ Shuangshuang Chen, ${ }^{2 *}$ Mengting Yao, ${ }^{2}$ Xuesong Jiang, ${ }^{1 *}$ Qinghua Lu $u^{1,2 *}$

${ }^{1}$ School of Chemistry and Chemical Engineering, Frontiers Science Center for Transformative

Molecules, the State Key Laboratory of Metal Matrix Composites, Shanghai Jiao Tong University, Shanghai, 200240 China.

${ }^{2}$ School of Chemical Science and Engineering, Tongji University, Shanghai, 200092 China.

*E-mail: fjchenss@sjtu.edu.cn; ponygle@sjtu.edu.cn; qhlu@sjtu.edu.cn. 


\section{Discussion S1}

The calculation of critical temperature to trigger wrinkle formation in the bilayer system.

When the bilayer was heated to $90{ }^{\circ} \mathrm{C}$ (close to the glass transition temperature of PSF) for 10 minutes, the innerstress between PSF skin layer and PDMS substrate was relieved after thermal relaxation. Upon cooling, compressive stress $\sigma$ was generated because of mismatched resilience and incoordinate surface area between the soft PDMS matrix and the hard PSF layer. According to linear bucking theory, ${ }^{1,2}$ the critical strain $\left(\varepsilon_{\mathrm{c}}\right)$ to trigger wrinkle formation in bilayer system can be calculated via Equation S1-S2:

$$
\begin{gathered}
\bar{E}=\frac{E}{1-v^{2}} \\
\varepsilon_{C}=\frac{1}{4}\left(\frac{3 \overline{E_{P D M S}}}{\overline{E_{P S F}}}\right)^{\frac{2}{3}}
\end{gathered}
$$

$\bar{E}, E$ and $v$ represent the in-plane modulus, Young's modulus and Poisson's ratio, respectively. $\mathrm{E}_{\mathrm{PDMS}}=2 \mathrm{MPa}, \mathrm{E}_{\mathrm{PSF}}=510 \mathrm{MPa}, v_{\mathrm{PDMS}}=v_{\mathrm{PSF}}=0.5$. So the resulting critical strain $\left(\varepsilon_{c}\right)$ is $1.3 \%$.

The applied strain $(\varepsilon)$ generated between PSF and PDMS was calculated via Equation S3:

$$
\varepsilon=\left(\alpha_{P D M S}-\alpha_{P S F}\right) \times \Delta T
$$

$\varepsilon, \alpha$, and $\triangle \mathrm{T}$ refer to applied strain, the linear thermal expansion coefficients and the temperature variation, respectively. Since $\alpha_{P D M S} » \alpha_{P S F}$, Equation S3 can be simplified as Equation $\mathrm{S} 4$, where $\alpha_{P D M S} \approx 30 \times 10^{-5} /{ }^{\circ} \mathrm{C}$.

$$
\varepsilon=\alpha_{s} \times \Delta T
$$

By calculation, the corresponding critical temperature $\left(T_{c}\right)$ was $46.7{ }^{\circ} \mathrm{C}$. In other words, wrinkles were formed when temperature was below $46.7^{\circ} \mathrm{C}$ and disappeared when higher than $46.7^{\circ} \mathrm{C}$. 


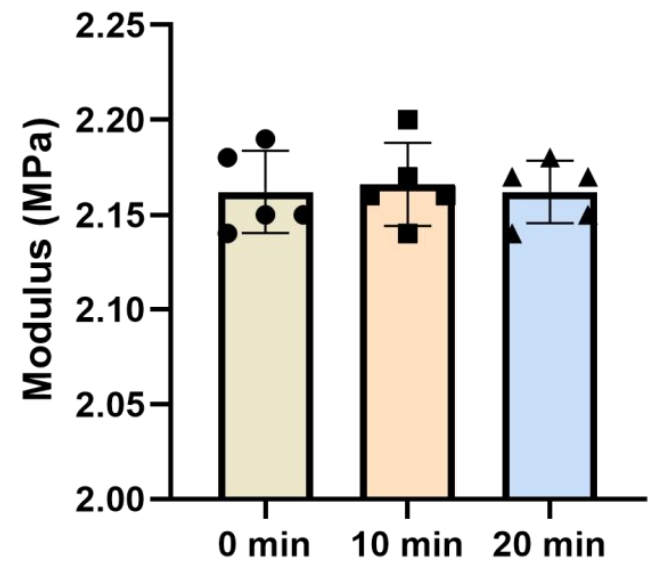

Figure S1. The modulus of elasticity of PDMS. The samples were measured with atomic force microscopy (AFM, MFP-3D) after heating to $90{ }^{\circ} \mathrm{C}$ for 0,10 or $20 \mathrm{~min}$, respectively, and then cooling to RT. Measuring parameters: spring constant $350.00 \mathrm{pN} / \mathrm{nm}$, DMT model, radius 5 nm.
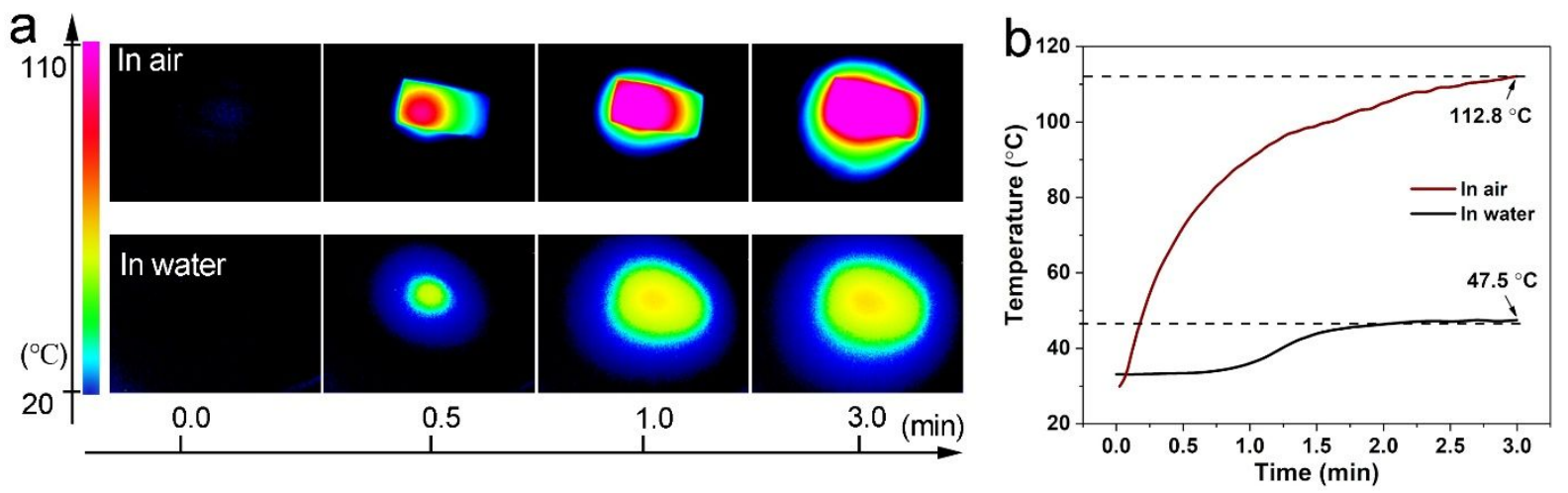

Figure S2. (a) NIR thermal images of bulk bilayers under NIR irradiation in the air (up) and PBS solution (bottom). NIR at $3.5 \mathrm{~W} \mathrm{~cm}^{-2}$ and $808 \mathrm{~nm}$. (b) The corresponding temperature during NIR irradiation.

Table S1. The heating speed and plateau temperature modulated via changing the contents of CNTs and powder of NIR, respectively. 
Supporting Information

\begin{tabular}{|c|c|c|c|c|c|c|c|c|}
\hline \multirow{2}{*}{$\begin{array}{l}\text { Power of NIR } \\
\left(\mathrm{W} \mathrm{cm}^{-2}\right)\end{array}$} & \multirow{2}{*}{$\begin{array}{c}\text { Contents of CNTs } \\
(\%)\end{array}$} & \multicolumn{7}{|c|}{ Temperature $\left({ }^{\circ} \mathrm{C}\right)$} \\
\hline & & $0 \mathrm{~min}$ & $0.5 \mathrm{~min}$ & $1 \mathrm{~min}$ & $1.5 \mathrm{~min}$ & $2 \min$ & $2.5 \mathrm{~min}$ & $3 \mathrm{~min}$ \\
\hline \multirow{3}{*}{2.5} & 0.015 & 22.0 & 40.4 & 45.3 & 47.3 & 48.7 & 49.6 & 50.3 \\
\hline & 0.025 & 22.0 & 47.6 & 56.1 & 60.6 & 63.5 & 65.3 & 66.7 \\
\hline & 0.050 & 22.0 & 58.8 & 70.8 & 74.5 & 79.4 & 80.9 & 81.9 \\
\hline \multirow{3}{*}{3.5} & 0.015 & 22.0 & 50.2 & 60.0 & 64.7 & 67.0 & 68.9 & 70.2 \\
\hline & 0.025 & 22.0 & 61.9 & 76.2 & 82.7 & 87.4 & 90.9 & 92.6 \\
\hline & 0.050 & 22.0 & 71.4 & 90.4 & 99.3 & 105.7 & 109.5 & 112.1 \\
\hline
\end{tabular}
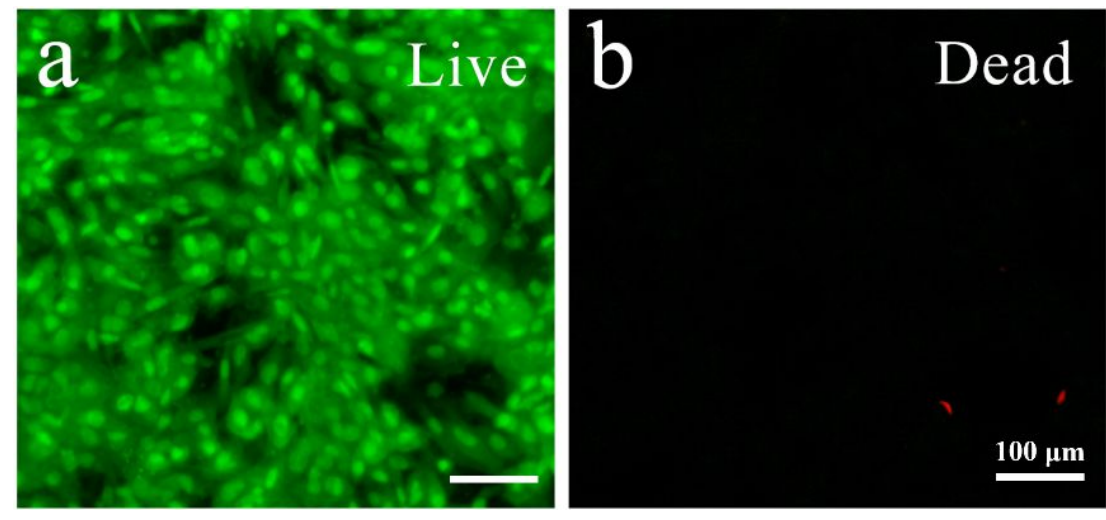

Figure S3. Fluorescence images of cell viability by AO/EB for fibroblast cells cultured on the surface of PSF. 


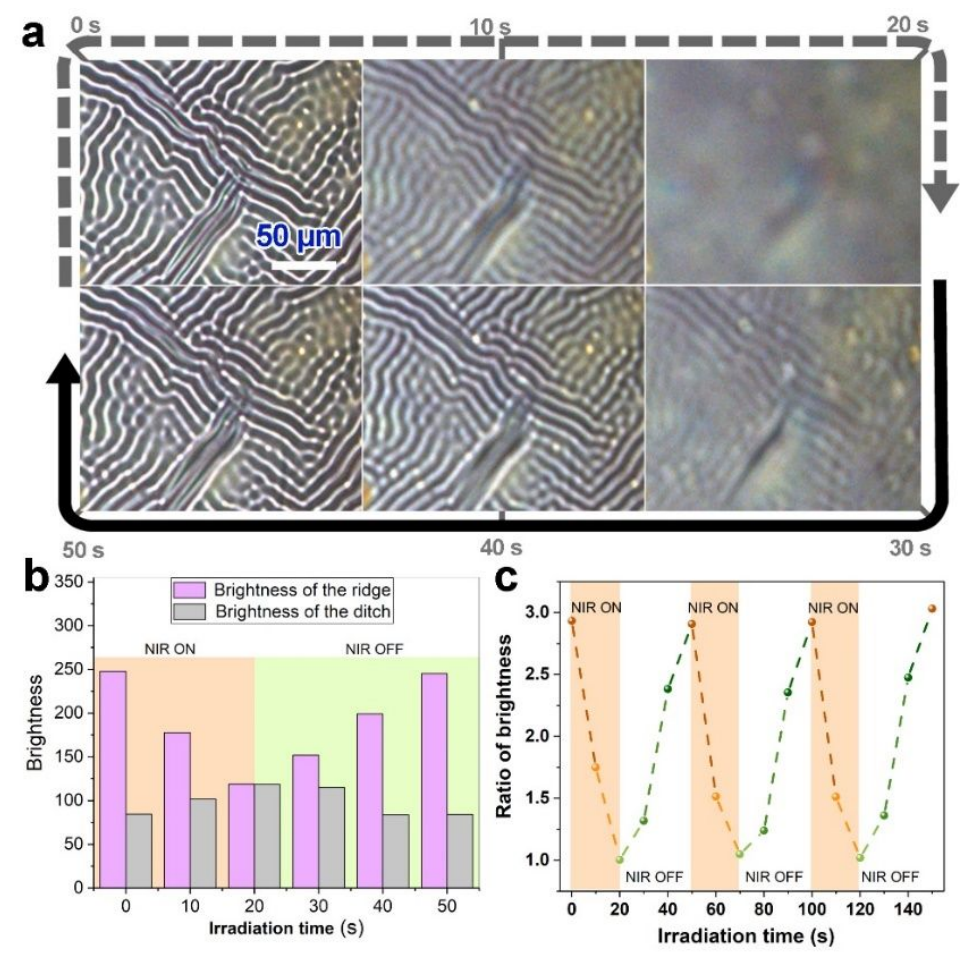

Figure S4. (a) Dynamic erasure/regeneration process of the PDMS/CNT/PSF wrinkle system in air during the on/off cycles of NIR light. (b) The evolution of the ratio of the brightness between the ridges and ditches in air during the on/off cycles of NIR light.The brightness of the ridges and ditches was recorded during the entire erasure/regeneration process. (c) The evolution of the ratio of the brightness between the ridges and ditches in air during the on/off cycles of NIR light.

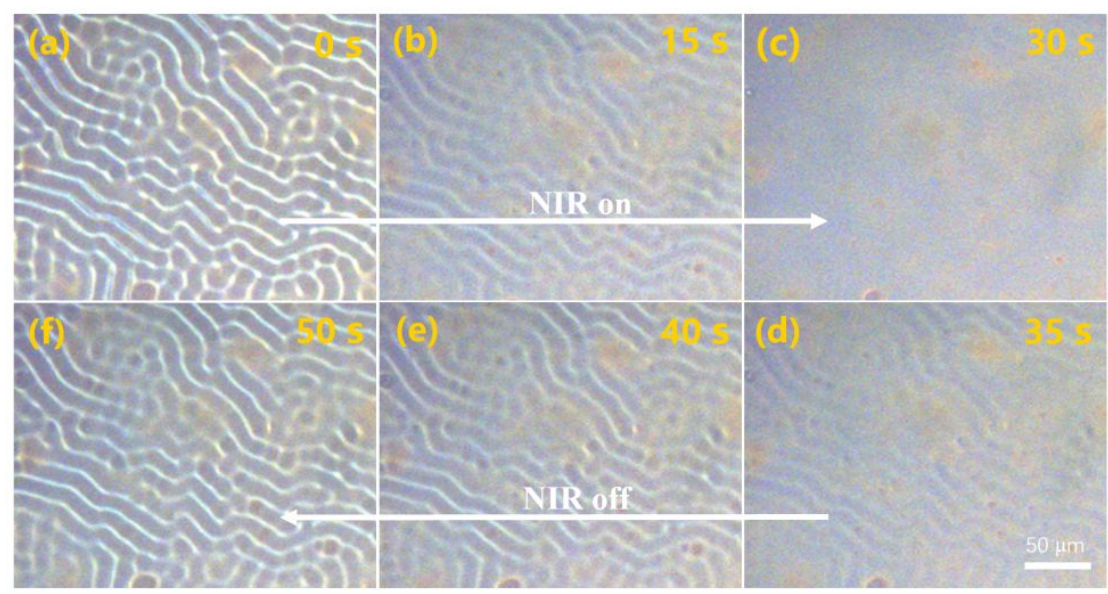

Figure S5. Dynamic erasure/regeneration process of a CNT-containing PDMS/PSF wrinkle system in PBS solution under 20s-on/5s-off cycles of NIR irradiation. (a-c) Optical images of 
the wrinkle erasure process by NIR irradiation. (d-f) Optical images of the wrinkle regeneration process after removal of NIR. The power and wavelength of NIR light were $3.5 \mathrm{~W} \mathrm{~cm}^{-2}$ and $808 \mathrm{~nm}$, respectively. The content of CNTs was $0.05 \%$. Scale bar $=50 \mu \mathrm{m}$.
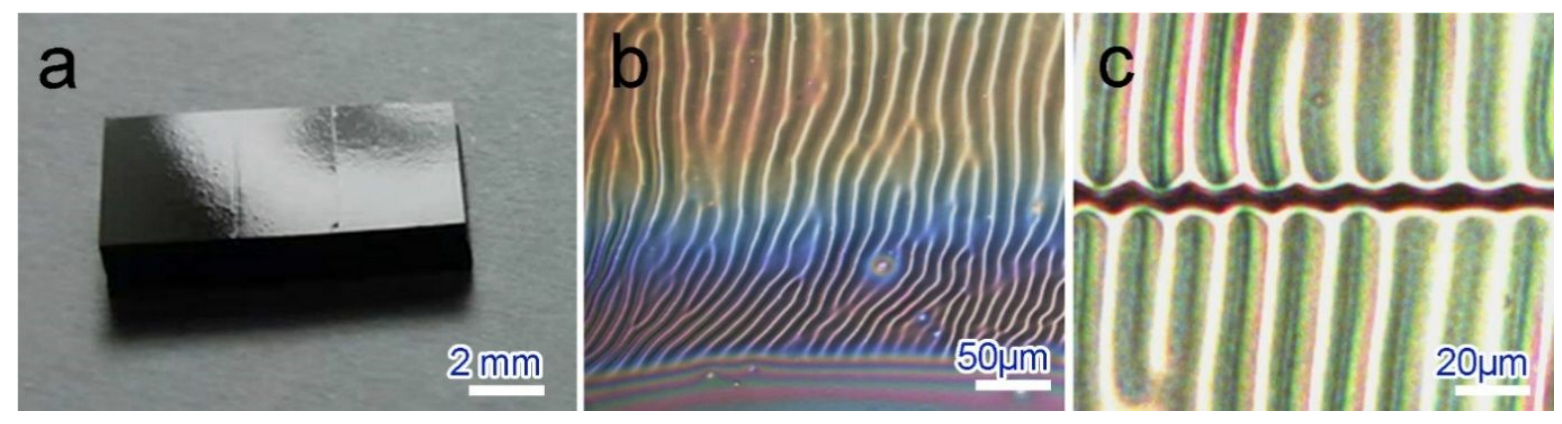

Figure S6. Ordered wrinkling patterns. (a) Optical images of PDMS-CNTs composite surface scratched with a medical surgical blade. (b) Wrinkle orientation perpendicular to the boundary of the sample. (c) Wrinkle orientation on both sides of the designed microgrooves.

Table S2. Comparison of the efficiency of cell sheet harvesting

\begin{tabular}{ccccc}
\hline Method & Cells & Time & Area & Ref \\
\hline Temperature-responsive & Oral mucosal epithelial cells & $>30 \mathrm{~min}$ & $1 \times 1 \mathrm{~cm}^{2}$ & 3 \\
\hline pH-responsive & Mesenchymal stem cells & $10-20 \mathrm{~min}$ & $1 \times 0.5 \mathrm{~cm}^{2}$ & 4 \\
\hline Magnetic manipulation & Different types & $>2$ days & None & 5 \\
\hline Photo-responsive & MC3T3-E1 cells & $>30 \mathrm{~min}$ & $<0.5 \times 0.5 \mathrm{~cm}^{2}$ & 6 \\
\hline Electro-responsive & Swiss 3T3 fibroblasts & $<10 \mathrm{~min}$ & $1 \times 1 \mathrm{~cm}^{2}$ & 7 \\
\hline This work & Fibroblast/different types & $<10 \mathrm{~min}$ & $1 \times 0.5 \mathrm{~cm}^{2}$ & \\
\hline
\end{tabular}

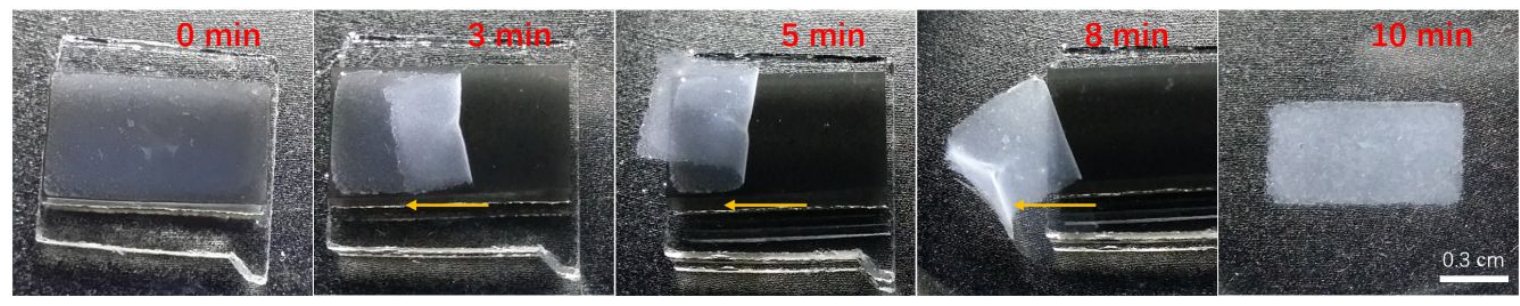


Figure S7. Disordered wrinkles for cell sheet harvesting at 0, 3, 5, 8, and 10 min, respectively. The power and wavelength of NIR light were $3.5 \mathrm{~W} \mathrm{~cm}^{-2}$ and $808 \mathrm{~nm}$. The content of CNTs was $0.05 \%$. Scale bar $=0.3 \mathrm{~cm}$.

Table S3. Table of average detachment times of non-wrinkle, ordered wrinkle (O-wrinkle) and disordered wrinkle (D-wrinkle) samples, time is shown in minutes. The efficiency of nonwrinkle was set as 100\%; the improvement in cell sheet harvesting using NIR-triggered ordered wrinkle and disordered wrinkle was quantitatively described.

\begin{tabular}{ccccccc}
\hline Sample & $20 \%$ & $40 \%$ & $60 \%$ & $80 \%$ & $100 \%$ & $\begin{array}{c}\text { Enhanced } \\
\text { efficiency }\end{array}$ \\
\hline Non-wrinkle & 4.0 & 6.0 & 8.0 & 10.5 & 14.5 & $100 \%$ \\
D-wrinkle & 3.0 & 4.5 & 6.0 & 8.0 & 10.0 & $131.0 \%$ \\
O-wrinkle & 2.5 & 3.5 & 5.0 & 6.5 & 8.5 & $141.4 \%$ \\
\hline
\end{tabular}

Note: The percentage was the ratio of released area to whole area for cell sheet.

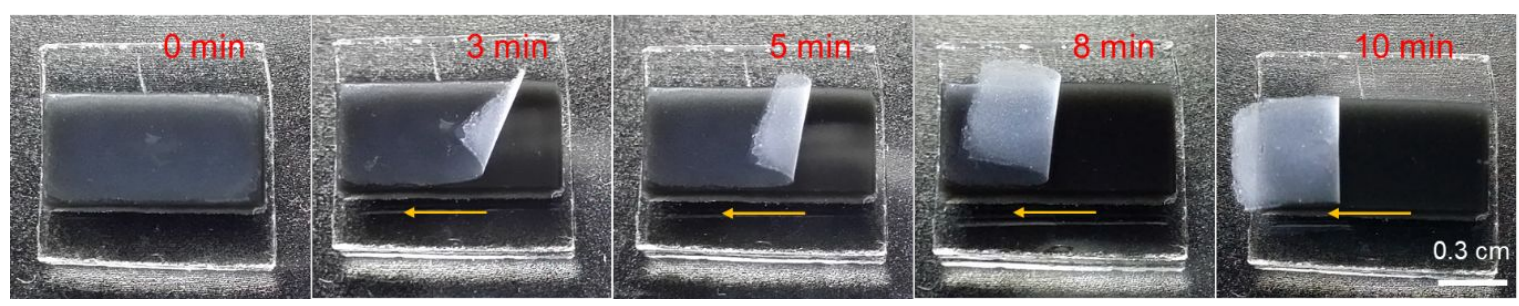

Figure S8. PDMS (non-wrinkle) samples for cell sheet harvesting at 0, 3, 5, 8, and $10 \mathrm{~min}$, respectively. The power and wavelength of NIR light were $3.5 \mathrm{~W} \mathrm{~cm}^{-2}$ and $808 \mathrm{~nm}$. The content of CNTs was $0.05 \%$. Scale bar $=0.3 \mathrm{~cm}$. 


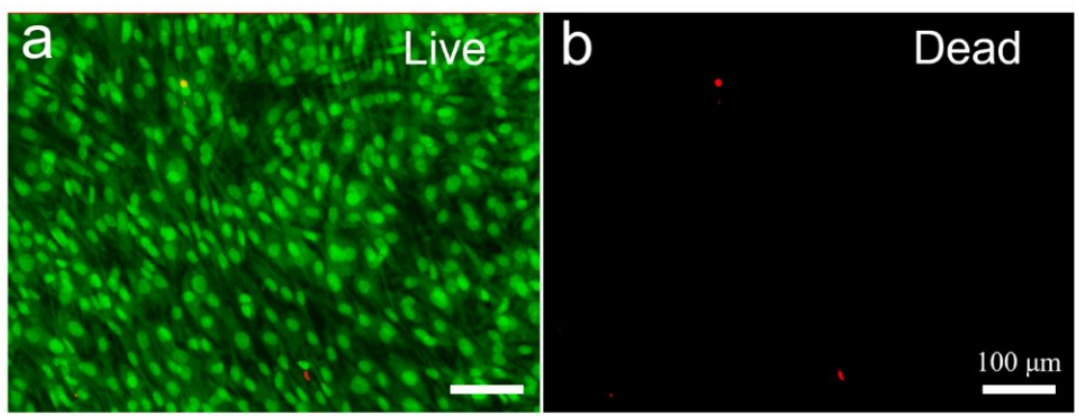

Figure S9. Fluorescence images of cell viability by AO/EB for a harvested fibroblast cell sheet.

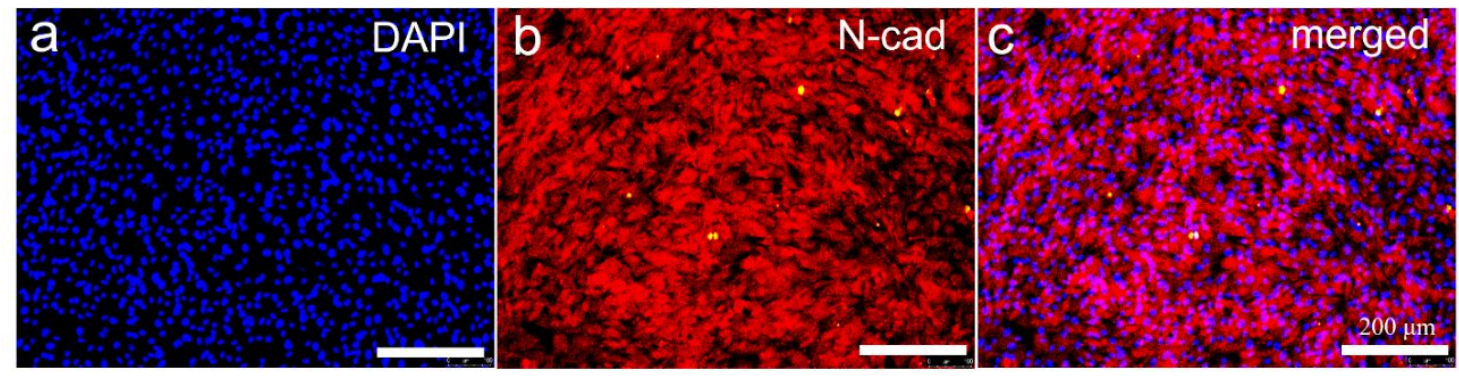

Figure S10. Immunofluorescence images of detached fibroblast sheet: DAPI (blue), N-cadherin (red) and merged image.
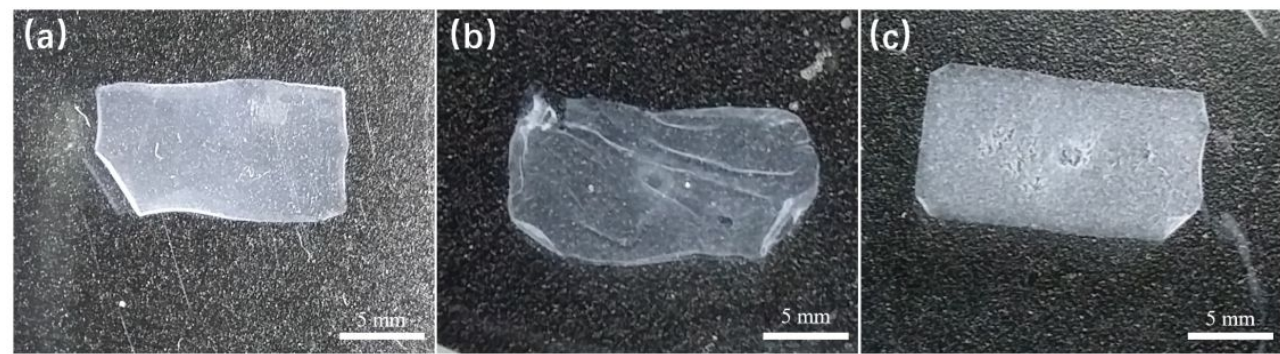

Figure S11. Optical images of whole detached cell sheets via dynamic wrinkles: (a) myoblast

C2C12 cell, (b) HeLa cell and (c) C6 cell. 


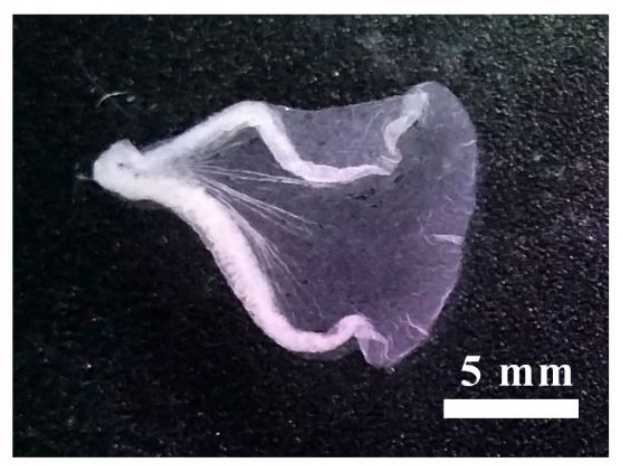

Figure S12. Cell sheet harvested from thermosensitive pNIPAm surfaces.
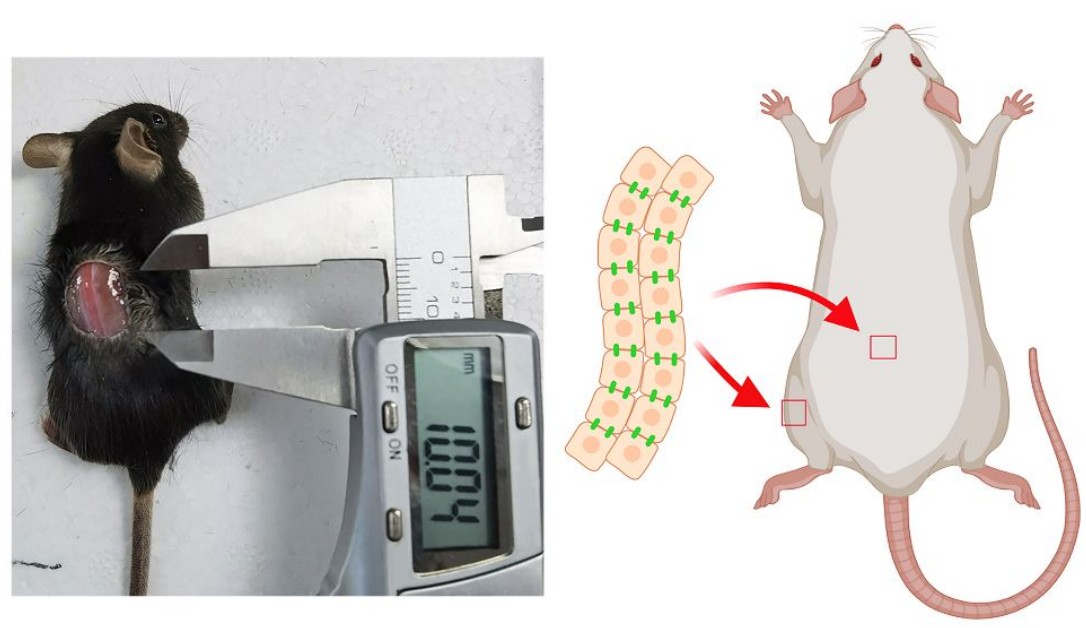

Figure S13. Experimental scheme showing the skin regeneration in mice by cell sheet transplantation.

Table S4. The wound closure rate for each group

\begin{tabular}{cccc}
\hline & Control & Wrinkle-Col Back & Wrinkle-Col Leg \\
\hline Day 1 & 0 & 0 & 0 \\
Day 3 & $18.9 \%$ & $35.7 \%$ & $47.1 \%$ \\
Day 6 & $39.7 \%$ & $62.6 \%$ & $82.1 \%$ \\
Day 8 & $43.9 \%$ & $74.6 \%$ & $95.3 \%$ \\
Day 10 & $61.3 \%$ & $81.9 \%$ & $100 \%$
\end{tabular}




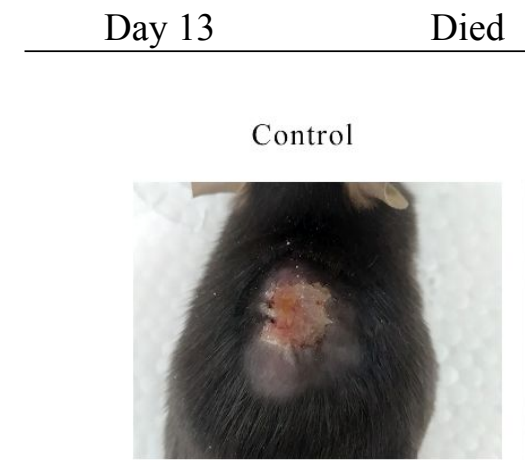

$61.3 \%$

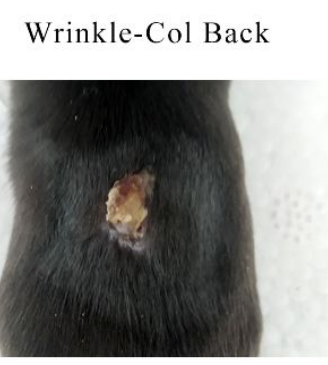

$81.9 \%$
pNIPAm Back

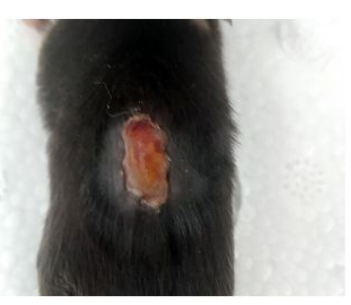

$64.2 \%$

Figure S14. Representative images of wound closure at $10^{\text {th }}$ day after cell sheet transplantation of each group (Control for no treatment of the wound, Wrinkle-Col Back and pNIPAm Back showed that cell sheets were harvested based on dynamic wrinkle and pNIPAm surfaces, respectively). The closure rates were calcultated compared to their freshly-cut wound area. 


\section{Supporting Movies}

Movie S1. Video of the dynamic wrinkle's erasure/regeneration process under the flicker of

NIR light in air.

Movie S2. Video of the dynamic wrinkle's erasure/regeneration process under the flicker of NIR light in PBS solution.

Movie S3. Video of the movement of polystyrene microspheres on the surface of dynamic wrinkle under the flicker of NIR light in PBS solution.

Movie S4. Video of cell sheet harvesting from the sandwich substrate.

\section{References}

(1) Li, F.; Hou, H. H.; Yin, J.; Jiang, X. S. Near-infrared light-responsive dynamic wrinkle patterns. Sci. $A d v$. 2018, 4.

(2) Yao, X.; Song, Y. L.; Jiang, L. Applications of Bio-Inspired Special Wettable Surfaces. Adv. Mater. 2011, 23, 719-734.

(3) Morino, T.; Takagi, R.; Yamamoto, K.; Kojima, H.; Yamato, M. Explant culture of oral mucosal epithelial cells for fabricating transplantable epithelial cell sheet. Regen Ther 2019, $10,36-45$.

(4) Guillaume-Gentil, O.; Semenov, O. V.; Zisch, A. H.; Zimmermann, R.; Voros, J.; Ehrbar, M. pH-controlled recovery of placenta-derived mesenchymal stem cell sheets. Biomaterials 2011, 32, 4376-4384.

(5) Zhang, W.; Yang, G.; Wang, X.; Jiang, L.; Jiang, F.; Li, G.; Zhang, Z.; Jiang, X. Magnetically Controlled Growth-Factor-Immobilized Multilayer Cell Sheets for Complex Tissue Regeneration. Adv. Mater. 2017, 29, 1703795.

(6) Yu, M. L.; Yu, M. F.; Zhu, L. Q.; Wang, T. T.; Zhou, Y.; Wang, H. M. The Effects of TiO2 Nanodot Films with RGD Immobilization on Light-Induced Cell Sheet Technology. Biomed Res. Int. 2015, 2015, 582359.

(7) Inaba, R.; Khademhosseini, A.; Suzuki, H.; Fukuda, J. Electrochemical desorption of selfassembled monolayers for engineering cellular tissues. Biomaterials 2009, 30, 3573-3579. 